\title{
A Feasibility Study for Usefulness of Preoperative Simulation of Medial Open-wedge High Tibial Osteotomy for Predicting Postoperative Realignment
}

\section{Shoji Konda}

Osaka University: Osaka Daigaku

\section{Teruya Ishibashi}

Osaka University: Osaka Daigaku

Masashi Tamaki

Osaka University: Osaka Daigaku

Kazuomi Sugamoto

Osaka University: Osaka Daigaku

Tetsuya Tomita ( $\square$ tomita@ort.med.osaka-u.ac.jp )

Osaka Daigaku Daigakuin Igakukei Kenkyuka Igakubu https://orcid.org/0000-0001-5291-7185

\section{Research Article}

Keywords: knee, osteoarthritis, medial OWHTO, preoperative surgical simulation, hinge axis

Posted Date: January 10th, 2022

DOI: https://doi.org/10.21203/rs.3.rs-1227060/v1

License: (c) (i) This work is licensed under a Creative Commons Attribution 4.0 International License.

Read Full License 


\section{Abstract}

Three-dimensional preoperative surgical realignment simulation of medial open-wedge high tibial osteotomy (OWHTO), in which simplified as the rigid rotation around the hinge axis, has been performed to predict the postoperative change and to develop a patient specific instrument for accurate osteotomy. However, the realistic practicality of this extremely simplified simulation method has not been verified. The purpose of this study was to investigate the usefulness of realignment simulation, in which medial OWHTO is simplified as a rotation around a hinge axis, in comparison with a postoperative CT model. Three-dimensional surface model of the tibia and femur was created from preoperative computedtomography (CT) images (preoperative model) of three patients. Sixty computer simulation models of the medial OWHTO in each patient were created by realignment simulation, in which medial OWHTO is simplified as the rigid rotation of proximal part of tibia relative to the distal part from 1 degree to 20 degrees around three type of hinge axes. The simulation models were compared with the actual postoperative model created from postoperative $\mathrm{CT}$ images to assess the reality of the simulation model. After the distal parts of the tibia between each simulation model and postoperative CT model were aligned by a surface registration, average surface distance between two models was calculated as an index representing the similarity of the simulation model to the postoperative model. The minimum average surface distance between the simulation and postoperative CT models were almost $1 \mathrm{~mm}$ in each patient. The rotation angles at which the minimum average surface distance was represented were almost identical to the actual correction angles. Overlaying the simulation and the postoperative CT models, we found that the posterior tibial tilt and the axial rotation of the proximal tibia of the simulation model well represented that of the postoperative CT model as well as the valgus correction. Therefore, the realignment simulation of medial OWHTO simplified as the rigid rotation around the hinge axis can generate the realistic candidates of postoperative realignment that includes the actual postoperative realignment, suggesting the usefulness for the preoperative simulation method.

\section{Background}

Medial open-wedge high tibial osteotomy (OWHTO) aims a realigning the femur and tibia[1]. In addition to the valgus correction, the control of posterior tibial slope (PTS) and internal/external rotation has been known as crucial factors associating with the postoperative result [2, 3]. Three-dimensional (3D) preoperative surgical realignment simulation of medial OWHTO has been performed to predict the postoperative change in the PTS and internal/external rotation as well as the valgus correction. In addition, 3D preoperative surgical realignment simulation has been used to develop a patient specific instrument for accurate osteotomy $[4,5]$. For predicting the change in the intra-articular load after medial OWHTO, the muscle skeletal simulation has been performed with the use of postoperative realignment simulation model[6, 7]. The postoperative realignment simulation of medial OWHTO has been performed with the use of preoperative 3D computed tomography (3D-CT) images and 3D surface model.

In postoperative realignment simulation of medial OWHTO, the position and orientation of the hinge axis and the osteotomy plane through that axis are defined, and the postoperative realignment is predicted by 
cutting the model at the osteotomy plane and realigning it around the hinge axis $[2,8,9]$. By combining the hinge axis and the rotation angle around the axis, it is possible to systematically create a variety of postoperative simulation models. However, the realistic practicality of this extremely simplified simulation model of a rotation axis and rotation around that axis has not been verified. To verify whether the virtual realignment generation realistically reflects the postoperative realignment, it is considered necessary to compare it with the actual postoperative realignment. Therefore, the purpose of this study was to investigate the usefulness of realignment simulation, in which medial OWHTO is simplified as a rotation around a hinge axis, in comparison with a postoperative CT model.

\section{Methods}

The present study was approved by the institutional research ethics committee, and all participants provided written informed consent. A male patient $(75 \mathrm{yrs} ., 167.0 \mathrm{~cm}, 69 \mathrm{~kg})$ and two female patients (71 yrs. $151.2 \mathrm{~cm}, 50.8 \mathrm{~kg}$ and $69 \mathrm{yrs} .146 \mathrm{~cm}, 72 \mathrm{~kg}$ ) who were scheduled medial OWHTO were included in this study. CT images of the three patients were acquired using a 3D-CT imaging system (Optima CT660Pro Advance; GE Healthcare, Milwaukee, WI), and the images were transferred to a 3D image analysis workstation (Volume Analyzer Synapse Vincent, Fujifilm Corporation, Tokyo, Japan). The contours of the cortical bone of the tibia, fibula, and femur were segmented semi-automatically from the CT images. A 3D surface model of the bones was reconstructed from the segmented images (preoperative CT model) (Figure 1a).

Three anatomical landmarks of the tibia (insertion of the anterior cruciate ligament and posterior cruciate ligament, and the center of the distal articular surface) were identified on the preoperative CT model to define the tibial anatomical coordinate system $[10,11]$. The preoperative CT model of the tibia and femur was transformed to the tibial coordinate system for the spatial normalization. Five additional landmarks were identified to determine the cutting plane (Figure 1b) and three axes of rotation (hinge axes) were defined (Figure 1c). The variations of the hinge axis can represent the clinically observed type of osteotomy [12]. The variations are set within the "safe-zone" of medial OWHTO [13]. Vertices of the preoperative CT model located above the osteotomy plane were translated by the matrix transformation which determined by the rotation angle $(\theta)$ around the hinge axis. In total, 60 simulations of the medial OWHTO in each patient were obtained with a combination of the 20 rotation angles and three hinge axes. The proximal part of tibia of the simulation models showed antero-posterior tilting and internal-external rotation in addition to the valgus-varus rotation because the given axes of rotation were not along the antero-posterior axis of the tibia (Figure $2 b$ ).

The simulation models were compared with the actual postoperative model to assess the reality of the simulation of medial OWHTO simplified as the rotation around hinge axis. The postoperative tibial model was created with a semi-automatic segmentation from the postoperative CT images. The inserted metal fixation plate was deleted manually. The postoperative CT model was cut below the osteotomy plane where the shape is not changed before and after the medial OWHTO. A surface registration of the distal part of the tibia was performed to align the simulation models and postoperative CT models using Image 
Registration Tool Kit [14]. Afterwards, the surface distance of the proximal tibia between the simulation models and postoperative CT models was evaluated. Average surface distance was calculated on between each simulation model and postoperative CT model as an index representing the similarity of the simulation model to the postoperative model

\section{Results}

Figure 3 demonstrates the results of patient 1 who was underwent average correction angle by medial OWHTO (10.3 degrees by the postoperative x-ray evaluation). When the axis of rotation was set to axis 1 or axis2, the average surface distance was smallest when it was rotated by 10 degrees. The rotation angle was consistent with the postoperative correction angle recorded by the postoperative x-ray evaluation. On the other hand, when the axis of rotation was set to axis3, the average surface distance was smallest when it was rotated by 13 degrees (Figure 3-a). Graphics shows the overlay of the simulation model generated by three different hinge axes (wire model in colors) on the postoperative CT model. The rotation angle of the model displayed in the graphics was set to the angle with the smallest surface distance, and -2 degrees and +2 degrees to it. The simulation model representing the smallest surface distance in each hinge axis (center column) demonstrated that the valgus angle of tibial plane is more consistent with the postoperative CT model on the backward view (Figure 3-b). The simulation model -2 degrees and +2 degrees to it demonstrated the under (left column) and over correction (right column) of medial OWHTO (Figure 3-b). When compared within the model in the center column, the model generated by the axis 2 or axis 3 are more fitted to the postoperative CT model than the axis 1 in the axial rotation of the tibial plane (Figure 3-d).

Figure 4 demonstrates the results of patient 2 who was underwent relatively small correction angle by medial OWHTO (8.2 degrees by postoperative x-ray evaluation). The average surface distance was smallest when it was rotated by 7 or 8 degrees. The rotation angle was consistent with the postoperative correction angle recorded by the postoperative $x$-ray evaluation. The simulation model representing the smallest surface distance in each hinge axis (center column) demonstrated that the valgus angle of tibial plane is more consistent with the postoperative CT model on the backward view (Figure 4-b). When compared within the model in the center column, the model generated by the axis2 was more fitted to the postoperative CT model than the axis1 and axis3 in the posterior tibial slope (Figure 4-c) and axial rotation of the tibial plane (Figure 4-d).

Figure 5 demonstrates the results of patient 3 who was underwent relatively large correction angle by medial OWHTO (12.2 degrees by postoperative x-ray evaluation). The average surface distance was smallest when it was rotated by 8,9 , and 10 degrees around hinge axis 1,2 , and 3 , respectively. The rotation angle was not an exact consistent with the postoperative correction angle recorded by the postoperative x-ray evaluation. The simulation model representing the smallest surface distance in each hinge axis (center column) demonstrated that the valgus angle of tibial plane is more consistent with the postoperative CT model on the backward view (Figure 5-b). When compared within the model in the 
center column, the model generated by the axis2 was more fitted to the postoperative CT model than the axis1 and axis3 in the posterior tibial slope (Figure 5-c) and axial rotation of the tibial plane (Figure 5-d).

\section{Discussion}

We aimed to investigate the usefulness of realignment simulation, in which medial OWHTO is simplified as the rigid rotation around the hinge axis, in comparison with a postoperative CT model. The minimum average surface distance between the simulation model generated by the rotation around three hinge axes and postoperative CT model were almost $1 \mathrm{~mm}$. The rotation angle at which the minimum average surface distance was represented on each hinge axis was almost identical to the actual correction angle. Overlaying the 3D surface models of the simulation and the postoperative $\mathrm{CT}$, we found that the posterior tibial tilt (Figures 3, 4, 5-c) and the axial rotation of the proximal tibia (Figures 3, 4, 5-d) of the simulation model well represented that of the postoperative CT model as well as the valgus correction (Figures 3,4 , $5-b)$. Therefore, the realignment simulation of medial OWHTO simplified as the rigid rotation around the hinge axis can generate the realistic candidates of postoperative realignment that includes the actual postoperative realignment, suggesting the usefulness for the preoperative simulation method.

A major advantage of realignment simulation in which medial OWHTO is simplified as a hinge axis and rotation around that axis is a systematic generation of the candidates of the postoperative realignment. The 60 generated simulation models have great variability; however, a part of the simulation models may show an unrealistic realignment. The acceptable simulations in the 60 simulations were selected based on the clinical criteria for femorotibial alignment as shown in Figure 6-a. The medial and lateral edges of the articular surface of the proximal tibia were identified on the frontal view of the simulation model, and were defined as 0 and $100 \%$, respectively. A femorotibial alignment where the mechanical line (the line connecting the center of the hip joint center and center of the distal tibia) passes through $62.5 \%$, called Fujisawa point, has been recognized as a clinically ideal alignment [15]. In this study, the acceptable range was set at $\pm 5 \%(57.5-67.5 \%)$ from the Fujisawa point [16-18]. The arc passing through points $57.5 \%$ and $67.5 \%$ centered on the hip joint center was determined to evaluate passes of the mechanical line of the simulation models. The simulation models that passed within the arc were selected, suggesting the candidates of clinically feasible postoperative realignment (Figure 6-b). Additional advantage is that the simulation can generate the variation of postoperative realignment from a preoperative alignment of single patient. A large-scale database of virtual postoperative realignment can be developed when applied to many preoperative patient alignments, and it will contribute a virtual installation of the fixation plate in a preoperative planning and a development of new surgical devices.

A major disadvantage of this simulation method was that the simplified simulation ignored a deformability of the bone and a process of biplane medial OWHTO. The simulation model was created by the rigid rotation around the set hinge axis between the proximal and distal tibial parts. In actual intraoperative situations, the tibia can be deformed manually at the preserved cortex when inserting a fixation plate in addition to the rotation around the hinge axis. The vertices of the surface model located superior to the osteotomy plane were transformed along the rotation around the hinge axis; however, the 
tuberosity is preserved with the distal tibial parts in the biplane medial OWHTO. Although the present simulation models the medial OWHTO as the rigid rotation around the hinge axis, the simulation model represents well represented the postoperative CT model.

\section{Declarations}

\section{Ethics approval and consent to participate}

The study was conducted in accordance with the guidelines of the Declaration of Helsinki and approved by the Institutional Ethics Committee of Osaka University Hospital (19027-2).

\section{Consent for publication}

Not applicable

\section{Availability of data and materials}

The datasets used during the current study are available from the corresponding author on reasonable request

\section{Competing interests}

All authors declare no conflict of interest.

\section{Funding}

There is no funding for this research.

\section{Authors' contributions}

Conceptualization, S.K., T.I., and T.T.; Data acquisition, T.I., M.T., and T.T.; Data analysis, S.K., and T.I.; Interpretation, S.K., T.I., M.T., K.S., and T.T. Writing original draft, S.K. T.I., and T.T. All authors critically revised the report, commented on drafts of the manuscript, and approved the final report.

\section{Acknowledgements}

Not applicable

\section{References}

1. Lobenhoffer P, Agneskirchner JD. Improvements in surgical technique of valgus high tibial osteotomy. Knee surgery, sports traumatology, arthroscopy : official journal of the ESSKA. 2003;11:132-8.

2. Lee BH, Ha CW, Moon SW, Chang M, Kim HY, Park SH, et al. Three-dimensional relationships between secondary changes and selective osteotomy parameters for biplane medial open-wedge high tibial 
osteotomy. The Knee. 2017;24:362-71.

3. Jang KM, Lee JH, Park HJ, Kim JL, Han SB. Unintended Rotational Changes of the Distal Tibia After Biplane Medial Open-Wedge High Tibial Osteotomy. The Journal of arthroplasty. 2016;31:59-63.

4. Victor J, Premanathan A. Virtual 3D planning and patient specific surgical guides for osteotomies around the knee: a feasibility and proof-of-concept study. The bone \& joint journal. 2013;95-B:153-8.

5. Donnez M, Ollivier M, Munier M, Berton P, Podgorski JP, Chabrand P, et al. Are three-dimensional patient-specific cutting guides for open wedge high tibial osteotomy accurate? An in vitro study. Journal of orthopaedic surgery and research. 2018;13:171.

6. Kuriyama S, Watanabe M, Nakamura S, Nishitani K, Tanaka Y, Sekiguchi K, et al. Large medial proximal tibial angles cause excessively medial tibiofemoral contact forces and abnormal knee kinematics following open-wedge high tibial osteotomy. Clin Biomech. 2020;80:105190.

7. Kuriyama S, Morimoto N, Shimoto T, Takemoto M, Nakamura S, Nishitani K, et al. Clinical efficacy of preoperative 3D planning for reducing surgical errors during open-wedge high tibial osteotomy. Journal of orthopaedic research : official publication of the Orthopaedic Research Society. 2019;37:898-907.

8. Moon SW, Park SH, Lee BH, Oh M, Chang M, Ahn JH, et al. The Effect of Hinge Position on Posterior Tibial Slope in Medial Open-Wedge High Tibial Osteotomy. Arthroscopy. 2015;31:1128-33.

9. Teng Y, Mizu-Uchi H, Xia Y, Akasaki Y, Akiyama T, Kawahara S, et al. Axial But Not Sagittal Hinge Axis Affects Posterior Tibial Slope in Medial Open-Wedge High Tibial Osteotomy: A 3-Dimensional Surgical Simulation Study. Arthroscopy. 2021;37:2191-201.

10. Kusano M, Yonetani Y, Mae T, Nakata K, Yoshikawa H, Shino K. Tibial insertions of the anterior cruciate ligament and the anterior horn of the lateral meniscus: A histological and computed tomographic study. The Knee. 2017;24:782-91.

11. Tajima G, Nozaki M, Iriuchishima T, Ingham SJ, Shen W, Smolinski P, et al. Morphology of the tibial insertion of the posterior cruciate ligament. J Bone Joint Surg Am. 2009;91:859-66.

12. Ogawa $\mathrm{H}$, Matsumoto $\mathrm{K}$, Akiyama $\mathrm{H}$. The prevention of a lateral hinge fracture as a complication of a medial opening wedge high tibial osteotomy: a case control study. The bone \& joint journal. 2017;99B:887-93.

13. Han SB, Lee DH, Shetty GM, Chae DJ, Song JG, Nha KW. A "safe zone" in medial open-wedge high tibia osteotomy to prevent lateral cortex fracture. Knee surgery, sports traumatology, arthroscopy : official journal of the ESSKA. 2013;21:90-5.

14. Rueckert D, Sonoda LI, Hayes C, Hill DL, Leach MO, Hawkes DJ. Nonrigid registration using free-form deformations: application to breast MR images. IEEE transactions on medical imaging. 1999;18:71221.

15. Fujisawa Y, Masuhara K, Shiomi S. The effect of high tibial osteotomy on osteoarthritis of the knee. An arthroscopic study of 54 knee joints. Orthop Clin North Am. 1979;10:585-608.

16. Kuriyama S, Watanabe M, Nakamura S, Nishitani K, Sekiguchi K, Tanaka Y, et al. Classical target coronal alignment in high tibial osteotomy demonstrates validity in terms of knee kinematics and 
kinetics in a computer model. Knee surgery, sports traumatology, arthroscopy : official journal of the ESSKA. 2020;28:1568-78.

17. Dugdale TW, Noyes FR, Styer D. Preoperative planning for high tibial osteotomy. The effect of lateral tibiofemoral separation and tibiofemoral length. Clin Orthop Relat Res. 1992:248-64.

18. Miniaci A, Ballmer FT, Ballmer PM, Jakob RP. Proximal tibial osteotomy. A new fixation device. Clin Orthop Relat Res. 1989:250-9.

\section{Figures}




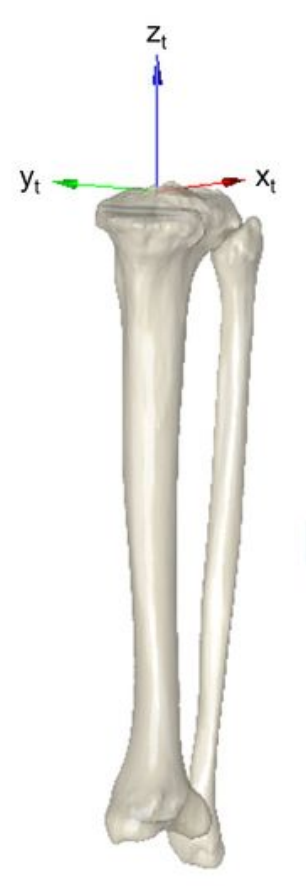

(a)

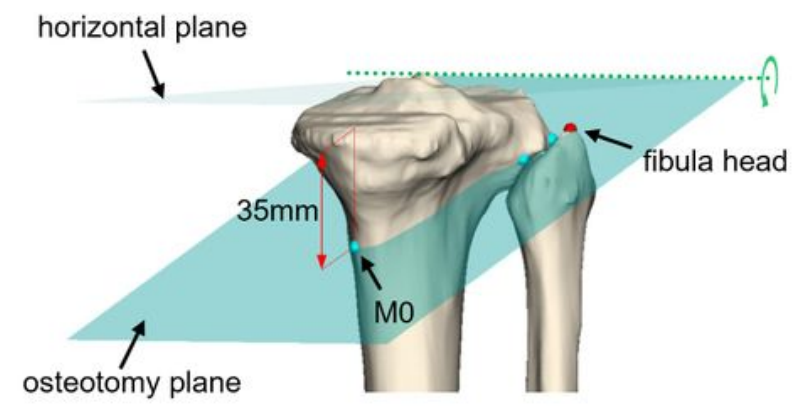

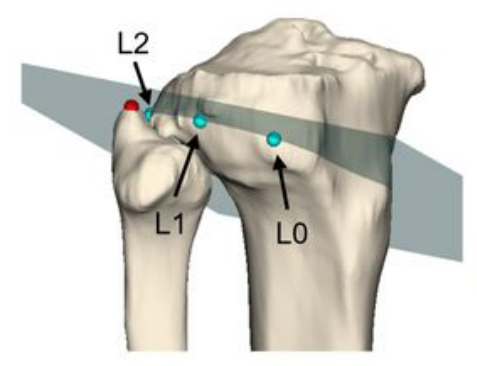

(b)

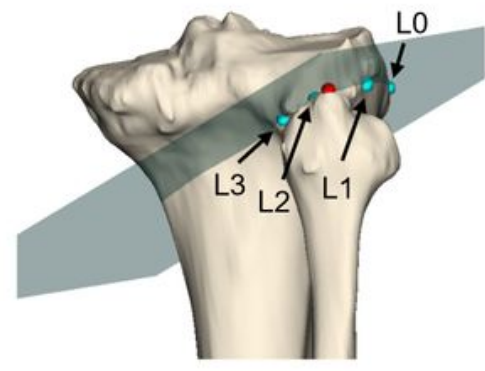

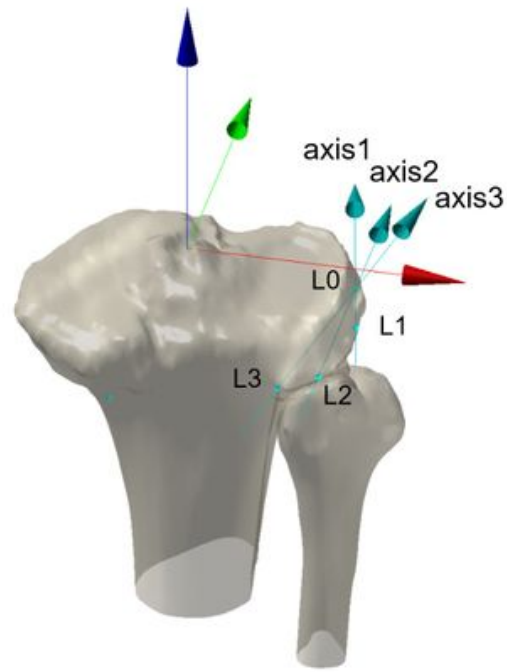

(c)

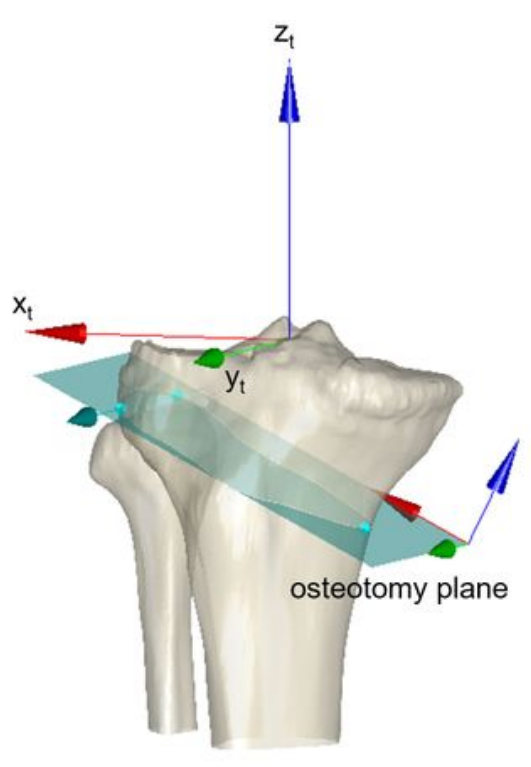

preoperative shape

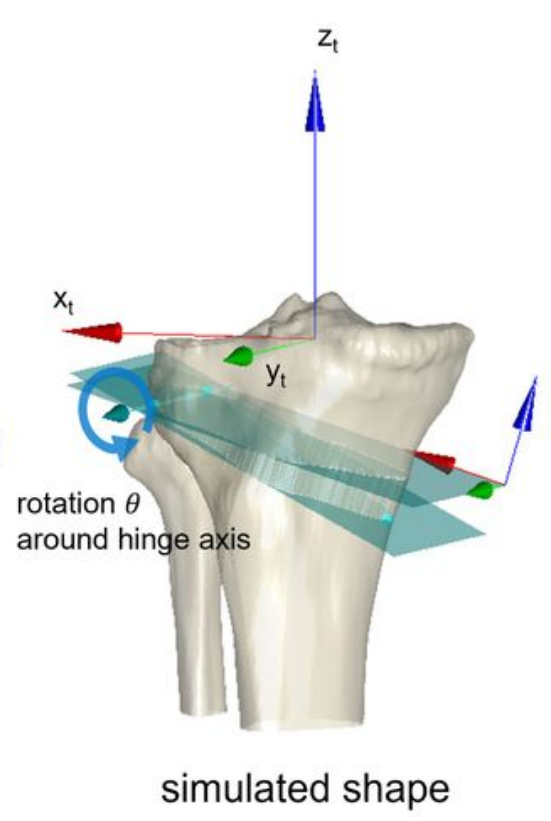

(d)

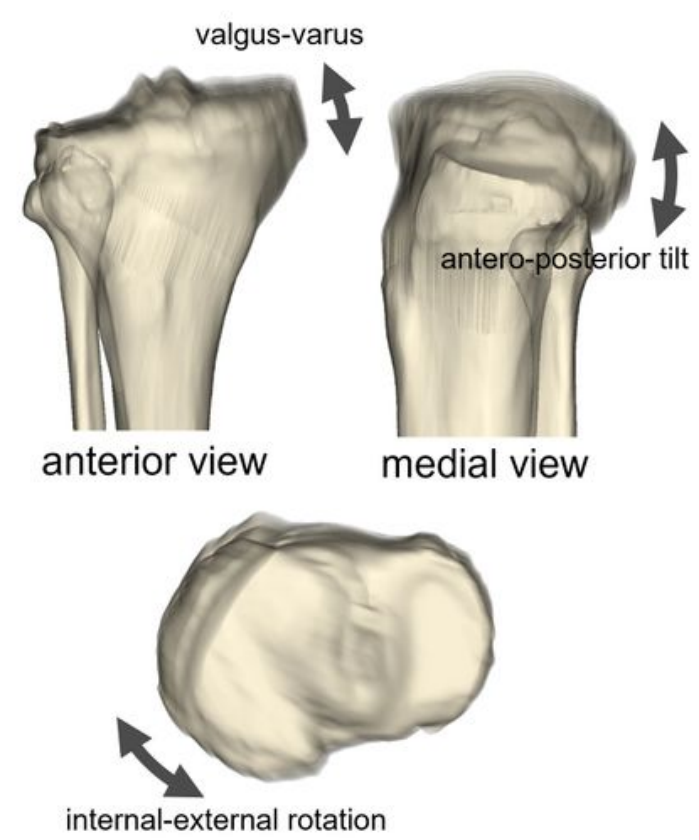

proximal view

(e)

\section{Figure 1}

Definition of bony landmarks and axis of rotation for realignment simulation. a) Preoperative 3D surface model was developed using CT images, and the preoperative CT-driven model was spatially normalized by the anatomical coordinate system. b) Cutting plane was defined by rotating horizontal plane around $y$ axis passing fibula head and M0 (35 mm inferior to the medical edge of articular surface). Additional landmarks (L0, L1, L2, and L3) were identified along with the cutting plane for simulating variation of 
clinically observed hinge axes in the medial OWHTO. d) Simulation of medial OWHTO rotating the proximal tibia around the given axis of rotation and (e) variation of 60 simulated shapes $(3$ axes $\times 20$ rotation angles) of the proximal tibia.

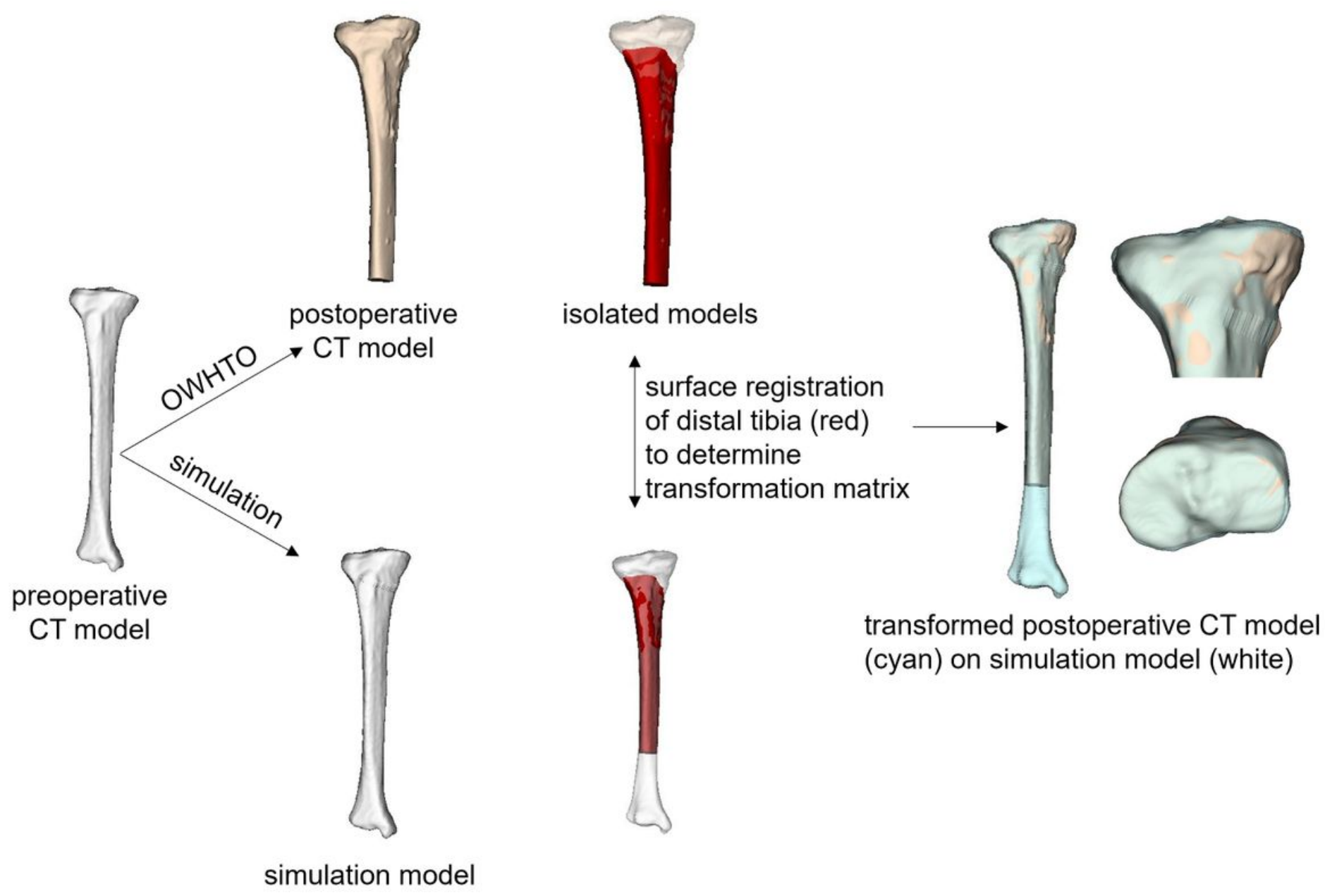

\section{Figure 2}

Comparison of simulation model and postoperative CT model. After the simulation and postoperative CT models were aligned by a surface registration of distal part of tibia, average surface distance between two models was calculated as an index representing the similarity of the simulation model to the postoperative model. 

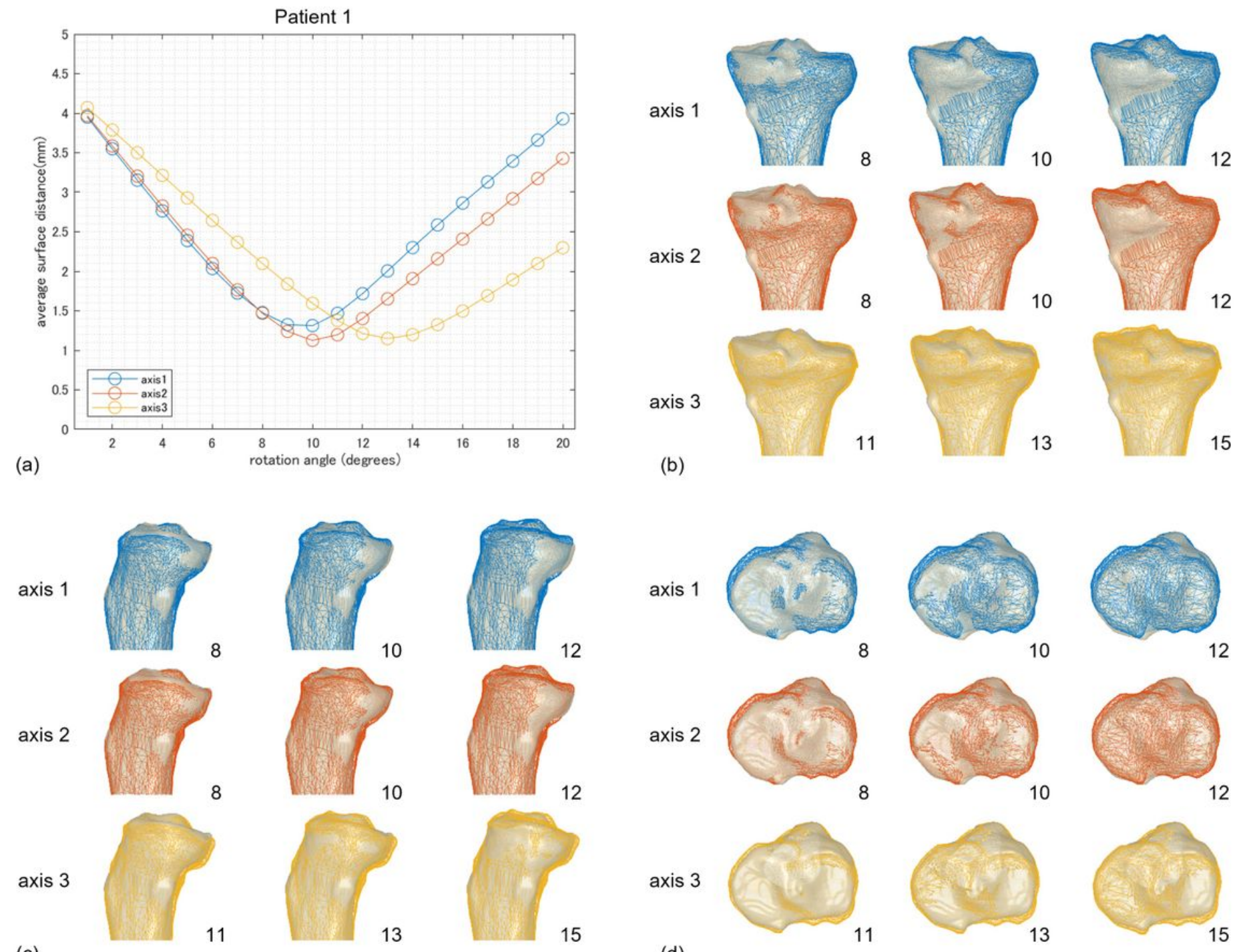

(b)
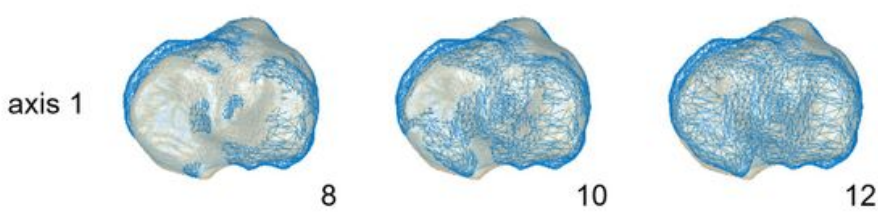

axis 2
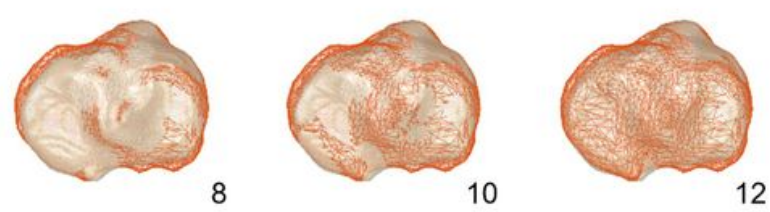

axis 3
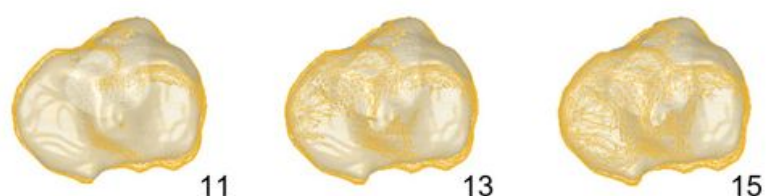

(d)

\section{Figure 3}

Results of patient 1 who was underwent average correction angle (10.3 degrees by the postoperative $\mathrm{x}$ ray evaluation). a) The average surface distance of each simulation model, in which generated by the combination of type of hinge axis and rotation angle, relative to the postoperative CT model. b, c, and d) Backward, medial, and proximal viewed images of overlay of the simulation model generated by three different hinge axes (wire model in colors) on the postoperative CT model. The number on the bottom right shows the rotation angle around each hinge axis. 

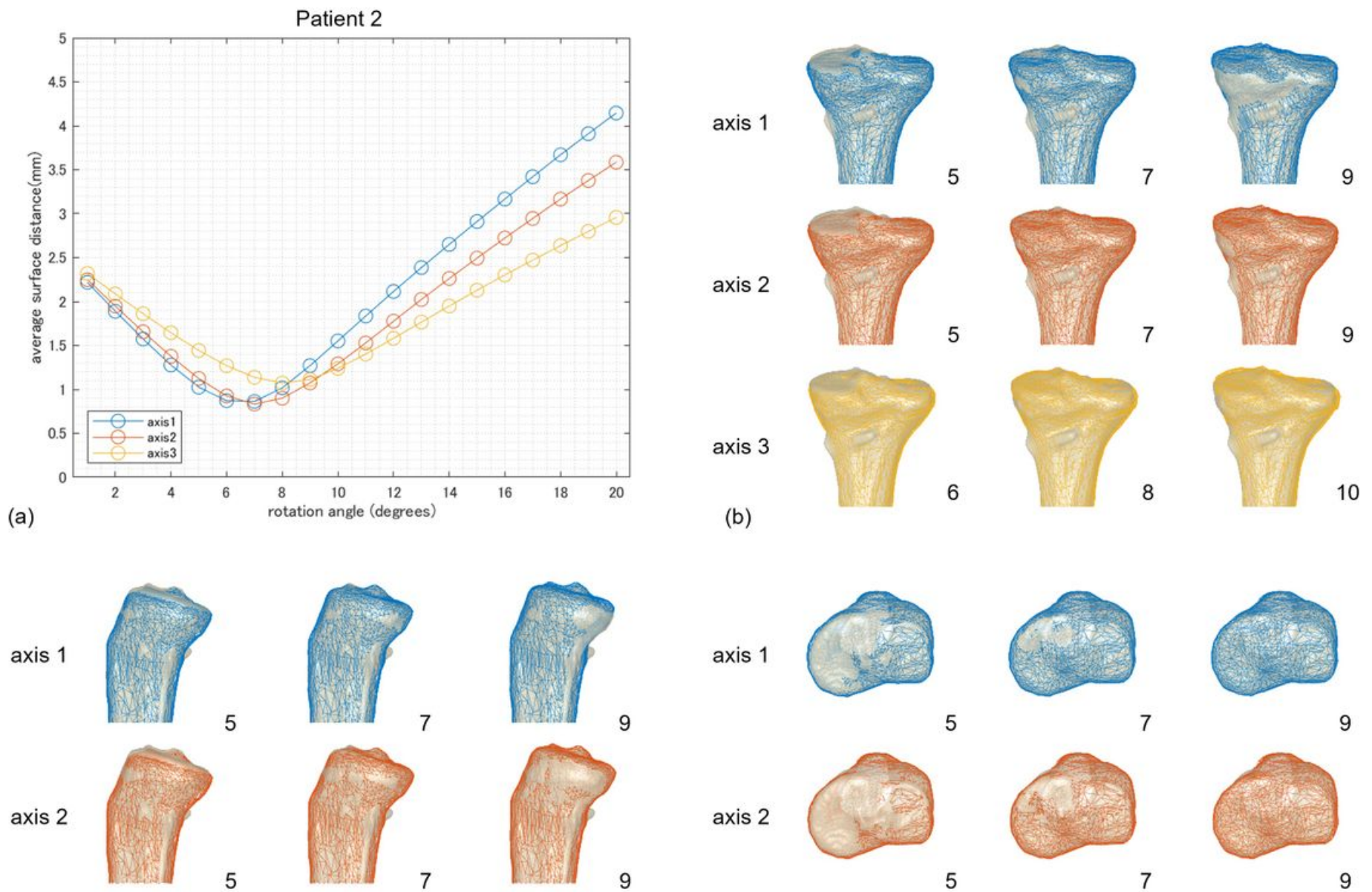

axis 3
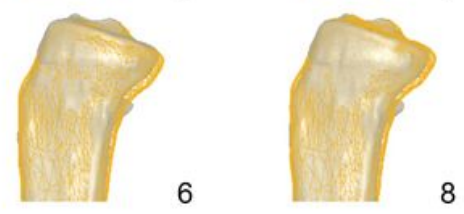

9

(c)

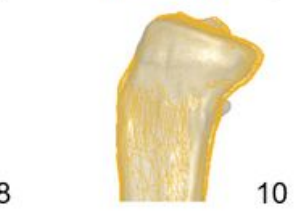

\section{Figure 4}

Results of patient 2 who was underwent relatively small correction angle ( 8.2 degrees by postoperative $x-$ ray evaluation). a) The average surface distance of each simulation model, in which generated by the combination of type of hinge axis and rotation angle, relative to the postoperative CT model. b, c, and d) Backward, medial, and proximal viewed images of overlay of the simulation model generated by three different hinge axes (wire model in colors) on the postoperative CT model. The number on the bottom right shows the rotation angle around each hinge axis. 


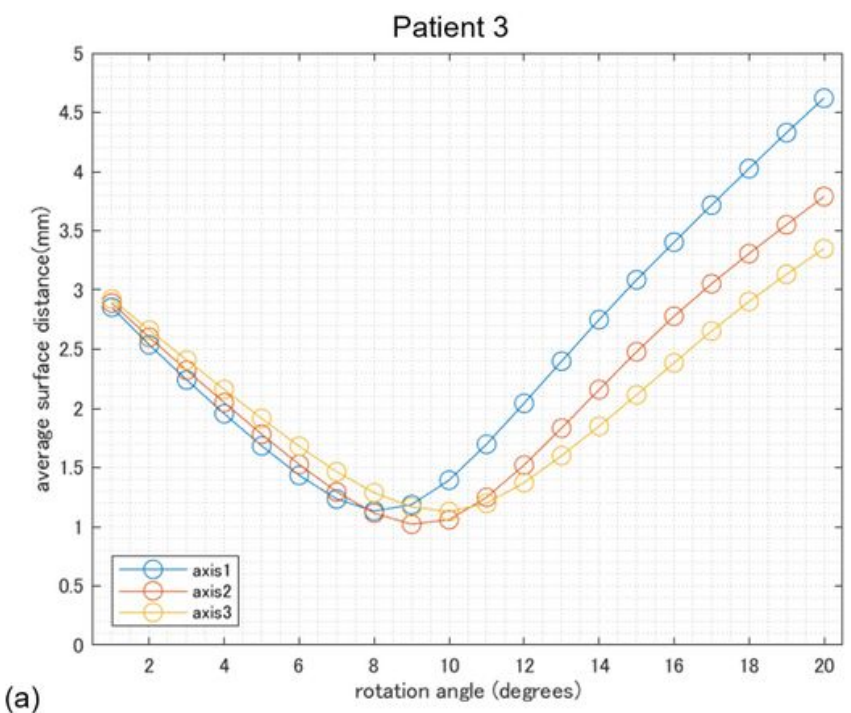

axis 1
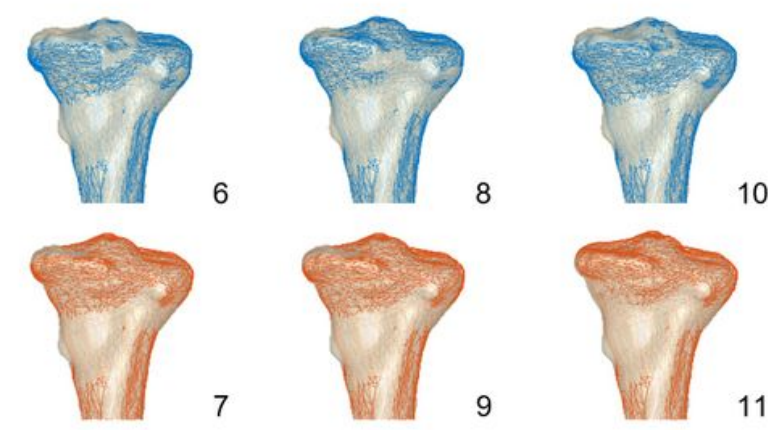

axis 2
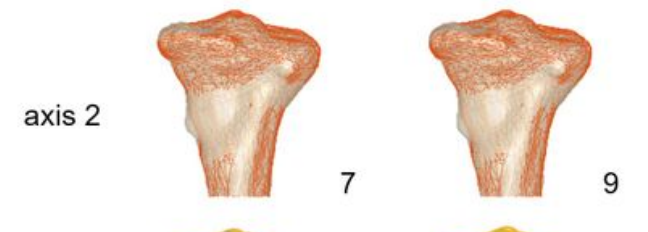

10

axis 3

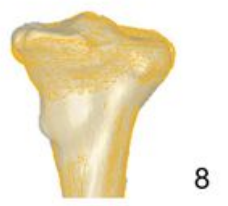

(b)
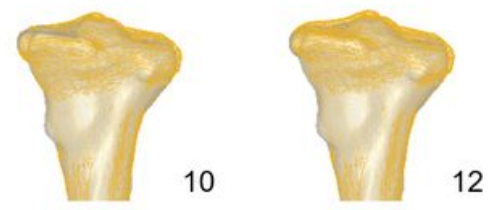

axis 1
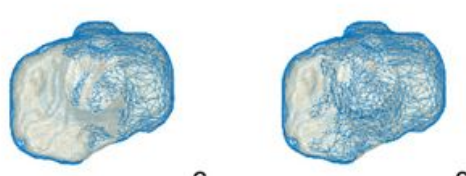

8

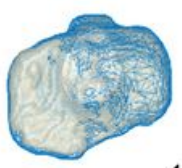

10

axis 2
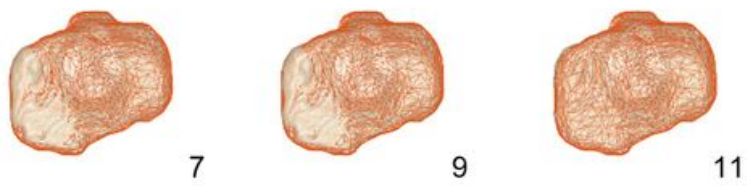

axis 3
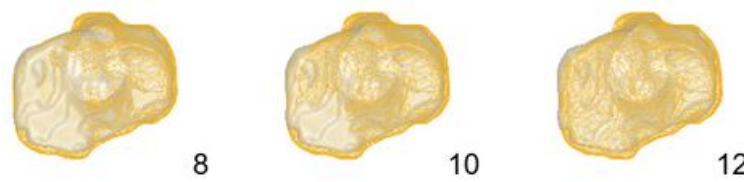

(d)

10

12

Figure 5

Results of patient 3 who was underwent relatively large correction angle (12.2 degrees by postoperative xray evaluation). a) The average surface distance of each simulation model, in which generated by the combination of type of hinge axis and rotation angle, relative to the postoperative CT model. b, c, and d) Backward, medial, and proximal viewed images of overlay of the simulation model generated by three different hinge axes (wire model in colors) on the postoperative CT model. The number on the bottom right shows the rotation angle around each hinge axis. 


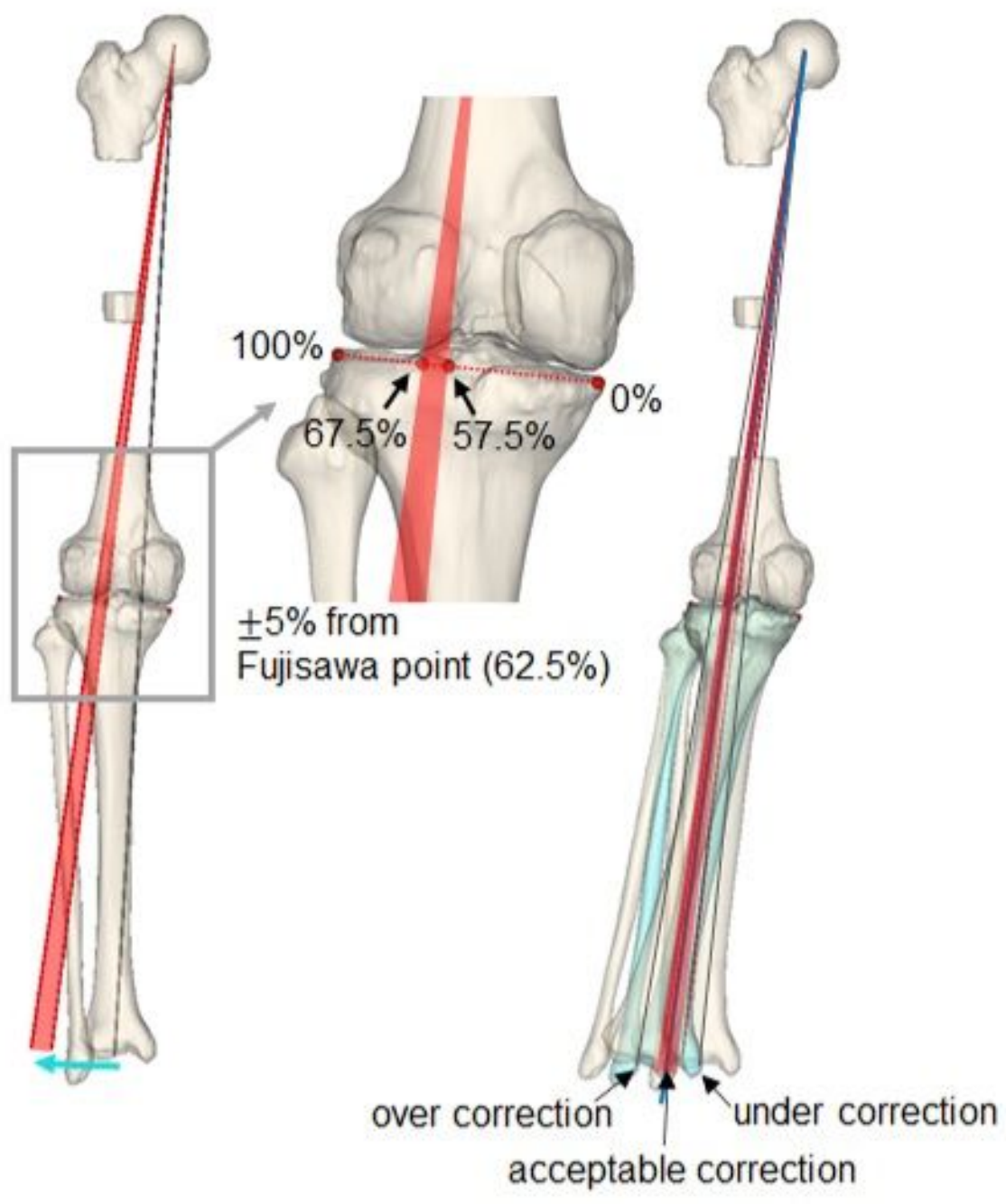

(a)

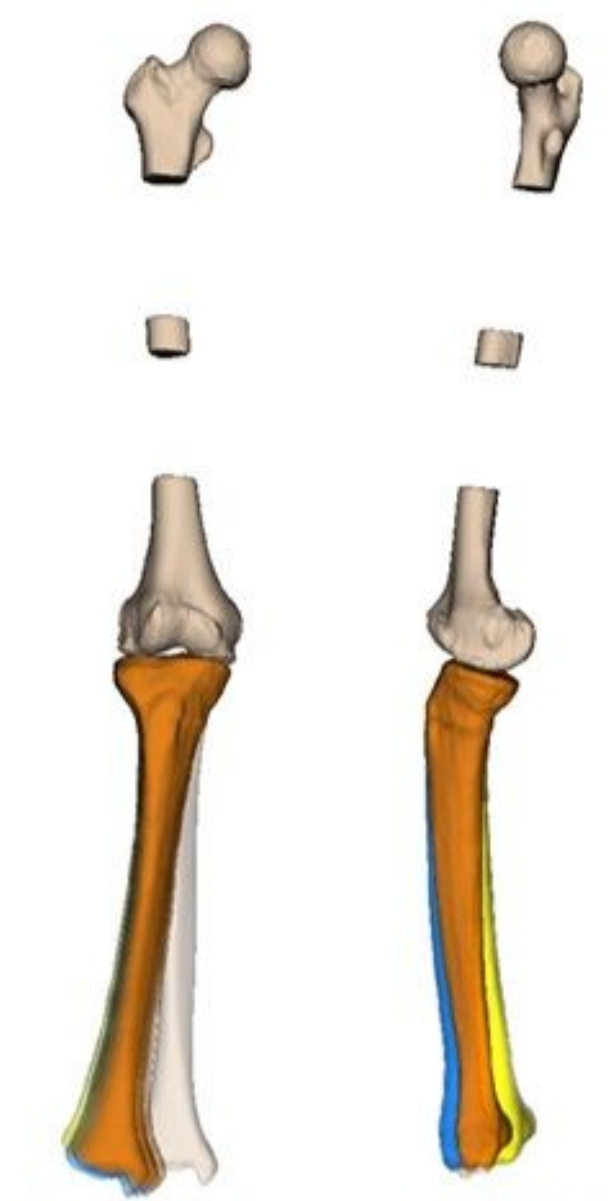

anterior view

medial view

\section{Figure 6}

Selection of clinically acceptable simulations and simulated postoperative realignment. (a) The gray line shows the preoperative mechanical line and red arc shows the clinically acceptable target alignment after the medial OWHTO. The simulation models which the mechanical line passes within the arc were selected as the clinically acceptable simulation. (b) Three-dimensional realignment variation of clinically acceptable simulation models with three type of hinge axes (axis 1: blue, axis 2: orange, axis 3: yellow). 